\title{
Evaluation of local - delivery system containing $80 \%$ aloe vera gel used as an adjunct to scaling and root planning in chronic periodontitis: A clinical study
}

\author{
Prakash Singh ${ }^{1}$ Manish Jain ${ }^{2}$, Vrinda Saxena ${ }^{3}$, Vijayta Sharva ${ }^{4 *}$, Meenakshi Boddun ${ }^{5}$ and Neha Jain $^{6}$ \\ ${ }^{1}$ Senior Lecturer, Public Health Dentistry, Bhopal, Madhya Pradesh, India \\ ${ }^{2}$ Hod \& Reader, Public Health Dentistry, Smbt Institute Of-422403 Dental Science \& Research, Igatpuri, Nashik, India \\ ${ }^{3}$ Proffesor \& Head, Public Health Dentistry,Gdc, Indore, Madhya Pradesh, India \\ ${ }^{4}$ Reader, Public Health Dentistry, Peoples Dental Academy, Bhopal, Madhya Pradesh, India \\ ${ }^{5}$ Reader, Department Of Periodontics, Peoples Dental Academy,Bhopal, Madhya Pradesh, India \\ ${ }^{6}$ Reader, Oral \& Maxillofacial Surgery, People’s Dental Academy, Bhopal, Madhya Pradesh, India
}

\begin{abstract}
Background: Recent interest and advances in the field of alternative medicine has promoted the use of various herbal and natural products for multiple uses in the field of medicine. Aloe vera is one such product exhibiting multiple benefits and has gained considerable importance in clinical research. This clinical study focuses on Aloe vera and highlights its property when used as a medicament in the periodontal pocket.
\end{abstract}

Aim: The aim of this study was Evaluation of local - delivery system containing $80 \%$ aloevera gel used as an adjunct to scaling and root planning in chronic periodontitis.

Objective: To evaluate the efficacy of Aloevera in reduction of gingivitis and periodontits and to compare the antioxidant level before and after alovera application on test site.

Material and method: Present study was conducted among 30 patients having periodontitis. Split mouth design was used. GI, SBI, CPI, LOA \& PD were recorded at baseline, 15 days $\&$ at 1 month. Immediately after recording all the indices at baseline and prior to drug application, the entire patient underwent scaling and root planning with hand instrument. The aloe vera gel was injected into the pockets with a syringe with blunt needle around the selected teeth in the treatment test site. The control site received only scaling and root planning. Biomechanical analysis-GCF was collected from $15 \mathrm{CP}$ patients before and after treatment. Total antioxidant status was determined using colorimetric method and commercial kits.

Result \& conclusion: In the present study there was a significant difference in antioxidant level in GCF between test site and control site. Test site showed significant increase in antioxidant level and significant improvement in clinical parameter 2 month after application of aloe vera along with scaling and root planning while on control site there was no significant change in antioxidant level. There was strong significant positive correlation of antioxidant with probing depth and attachment loss.

\section{Introduction}

Periodontal diseases are chronic infectious diseases characterized by a bacterial challenge that can provoke a destructive host response, leading to clinical attachment loss and ultimately possible tooth loss [1]. It is well established that supragingival plaque is the cause of gingivitis and plays a primary role in the initiation of periodontitis [2]. The removal of microbial plaque leads to resolution of gingival inflammation, and cessation of plaque control leads to a recurrence of inflammation [3]. It has been shown that rigorous self-performed plaque control over long periods of time reduced the levels and altered the composition of sub gingival bacteria and reduced the frequency of deep periodontal pockets [4].

The inability of the general adult population to perform adequate tooth brushing has led to the search for chemotherapeutic agents to improve plaque control [5] these chemicals, mainly triclosan and chlorohexidine, have been used as mouth rinses or added to dentifrices to avoid plaque formation and development of gingivitis [6]. Because some of these substances may have undesirable side effects, such as tooth staining and taste alteration, phototherapeutic agents with antimicrobial and anti-inflammatory properties have been investigated [7] The use of natural products in the prevention and treatment of oral conditions has increased recently and could be also beneficial in plaque control especially to urban and rural communities of low socioeconomic levels [8].

Aloe Vera is the most accepted species for various medical, cosmetic, and neutraceutical purposes [9]. The composition of Aloe Vera is complex. It consists of 75 different ingredients including

*Correspondence to: Vijayta Sharva, Reader, Public Health Dentistry, Peoples Dental Academy, Bhopal, Madhya Pradesh, India, Tel: 9827563977; E-mail: vijaytasharva@gmail.com

Key words: aloevera gel, gingivitis, periodontitis, antioxidants

Received: June 30, 2019; Accepted: July 15, 2019; Published: July 18, 2019 

study

vitamins, minerals, enzymes, sugars, anthraquinones or phenolic compounds, lignin, saponins, sterols, amino acids and salicylic acid [10]. The pharmacological actions of Aloe Vera gel as studied in vitro and in vivo include anti-inflammatory, antibacterial, antioxidant, immune-boosting and hypoglycaemic properties [11] Yagi et al [12] reported that Aloe Vera gel contains a glycoprotein with cell proliferating-promoting activity, while Davis et al [13] noted that Aloe Vera gel improved wound healing by increasing blood supply, which increased oxygenation as result.

Additionally, many authors reported that Mouth rinse and toothpaste [14] containing Aloe vera might be useful herbal formulations for chemical plaque control and improvement in plaque and gingival status in chronic gingivitis. Moreover, Pradeep et al [15] found that toothpaste containing Aloe vera was effective in reducing the microbial colonies of S. Sangius, S. Oralis, Actinomyces viscosus and Actinomyces naeslund in patients with chronic gingivitis. The above-mentioned properties, along with the ease of availability, no known adverse effects, and cost effectiveness, make Aloe vera an ideal candidate for plaque control, thereby reducing gingivitis and most likely eventual periodontitis. Hence, the aim of the present study is Evaluation of local - delivery system containing $80 \%$ aloevera gel used as an adjunct to scaling and root planning in chronic periodontitis.

\section{Material and methods}

The study design was a randomized, placebo-controlled clinical study for a period of three months. 30 subjects with plaque-induced gingivitis \& chronic periodontitis were included, within the ages of 18-65 years; they were systemically healthy individuals, had not undergone prophylaxis for the last six months, and were with at least 20 teeth present. Patients on antibiotics/anti-inflammatory therapy for the last six months, pregnant females, those with a history of allergy to aloe Vera, and patients unwilling to complete treatment were excluded from the study. Ethical clearance: was obtained from the institutional ethical committee(2015/002/RAC/2)(2015/200/IEC/2) and Informed written consent was taken from patient.

The questionnaire and data collection sheet were used for collection of desired information and to record the data at baseline, 1 month and 3 months interval. The data was collected by a single investigator. The following clinical parameters were being measured before and after treatment:

Probing pocket depth, Clinical attachment loss, Sulcus bleeding index, Superoxide dismutase, Total antioxidant status. All assessments were performed using standardized William's periodontal probe. PD Was measured in millimetres as the distance between the gingival margin and the base of the periodontal pocket. Attachment loss was also measured in millimeters as the distance between the cementenamel junction and the base of the gingival sulcus. both PD and CAL were evaluated four sites (mesio-buccal, mid-buccal, disto-buccal and lingual) per tooth.

This study was performed according to the split-mouth design in which the patients received two treatment modalities to the right or left half of the dentition. On the case side of the mouth

Full mouth supra-and-sub gingival scaling and root planning performed using ultrasonic and hand instruments to be followed by sub gingival delivery of aloe vera gel was injected into the pocket around the selected teeth. The pocket opening was covered with coe -pak. The control site received only scaling and root planning.
For Biomechanical analysis GCF was collected from 30 chronic periodontitis patients before and after treatment. Total antioxidant status was determined using colorimetric method and commercial kits.

GCF samples were collected from each patient ,using micro capillary tube, The samples were transferred from pipettes into small containers and immediately phosphate buffer saline was added to make a volume up to $3 \mathrm{ml}$ and stored at $-70^{\circ} \mathrm{C}$ for estimation of MDA level by colorimeter the sample then were send to laboratory for the assessment of MDA.

Aloe vera (Barbadensis Millar) gel was prepared by extraction process of aloe vera leaf, washing, trimming ,peeling was done then aloe vera extract $20 \mathrm{ml}$ was collected, The gel extraction from Aloe Vera leaves had been carried out by removing of its exudates and its mucilage was scraped out with blunt edged knife. This mucilage was stirred vigorously in a blender to make it uniform. This solution was strained through a muslin cloth and filtered. This uniform solution was extracted for cold- extracted gel (CEG) and hot extracted gel (HEG). Then hydroxy propyl methyl cellose $(1.5 \%=750 \mathrm{mg})$ was and $0.01 \%$ preservatives (methyl paraben + propyl paraben)+distilled water (5 $\mathrm{ml}$ ) was mixed. Aloe Vera gel was stored in the refrigerator in a plastic container that is safe for food storage or a glass container. Aloe vera gel was prepared by group of the faculty of pharmacy (People's Institute of Pharmacy and Research Center, Bhopal)

Statistical analysis was carried out using statistical package of social science (SPSS version 22) Student t test applied to compare between case and control site Repeated measure of ANOVA was apply to compare between time interval.

\section{Results}

Figure 1 reveals comparison of Probing pocket depth between case \& control site among chronic generalized periodontitis patients. At baseline and after 1 month of treatment there was statistically no significant difference in Probing pocket depth between case and control. After two month among case it reduced from $6.10 \pm 1.07$ to $3.00 \pm 0.79$ i.e. $50.81 \%$ reduction while among control it reduced from $5.60 \pm 1.23$ to $3.53 \pm 0.77$ i.e. $36.96 \%$ reduction. After two month there was significantly more reduction in PPD among cases as compare to control and there was statistically significant difference between case and control IN PPD ( $\mathrm{P}=0.039)$.

Figure 2 reveals comparison of Clinical Attachment loss between case \& control site among chronic generalized periodontitis patients. At baseline and after 1 month of treatment there was statistically no significant difference in clinical attachment loss between case and control. After two month among case it reduced from $6.93 \pm 0.85$ to $5.48 \pm 0.87$ i.e. $34.05 \%$ reduction while among control it reduced from $56.70 \pm 0.73$ to $5.27 \pm 0.91$ i.e. $21.34 \%$ reduction. After two month there was significantly more reduction in CAL among cases as compare to control and there was statistically significant difference between case and control in $\operatorname{CAL}(\mathrm{P}=0.001)$.

Figure 3 reveals comparison of Sulcus Bleeding Index between case \& control site among chronic generalized periodontitis patients. At baselinethere was statistically no significant difference in Probing pocket depth between case and control. After two month among case it reduced from $2.12 \pm 0.26$ to $0.90 \pm 0.41$ i.e. $57.54 \%$ reduction while among control it reduced from $2.27 \pm 0.24$ to $1.35 \pm 0.30$ i.e. $40.52 \%$ reduction. After two month there was significantly more reduction in SBI among cases as compare to control and there was statistically significant difference between case and control in SBI $(\mathrm{P}=0.001)$. 

study

Figure 4 reveals Comparison of Superoxide dismutase between case \& control site among chronic generalized periodontitis patients. At baseline there was statistically no significant difference in Superoxide dismutase between case and control. After two month among case

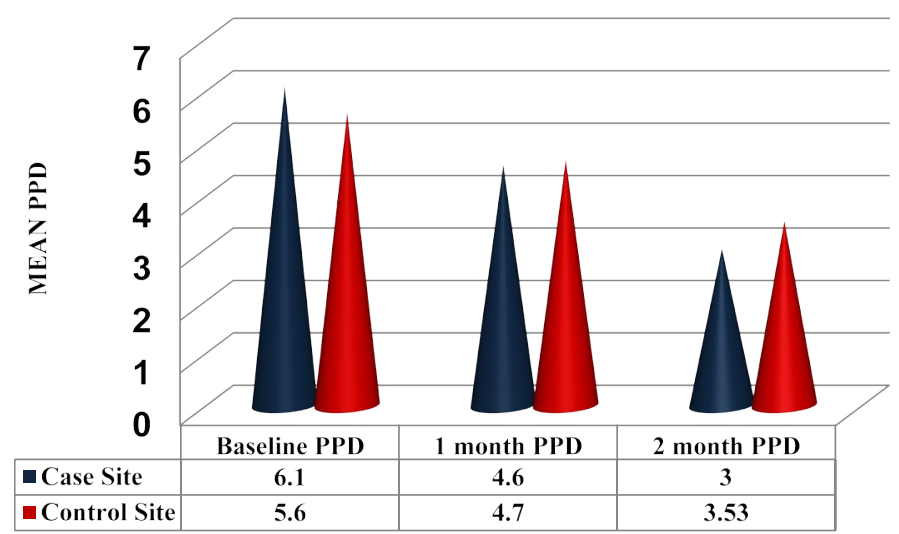

Figure 1. Comparison of Probing pocket depth between case \& control site among chronic generalized periodontitis patients

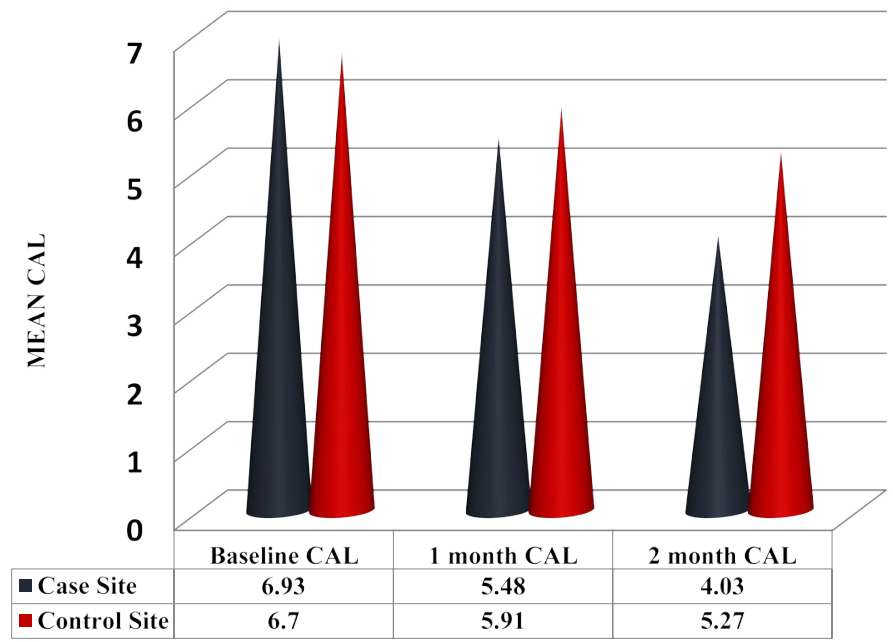

Figure 2. Comparison of Clinical Attachment loss between case \& control site among chronic generalized periodontitis patients

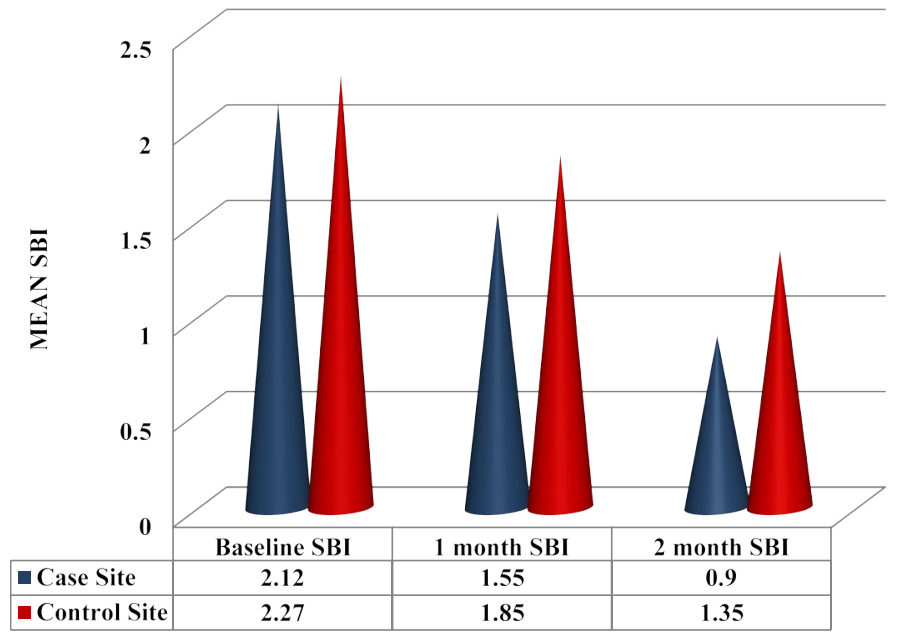

Figure 3. Comparison of Sulcus Bleeding Index between case \& control site among chronic generalized periodontitis patients

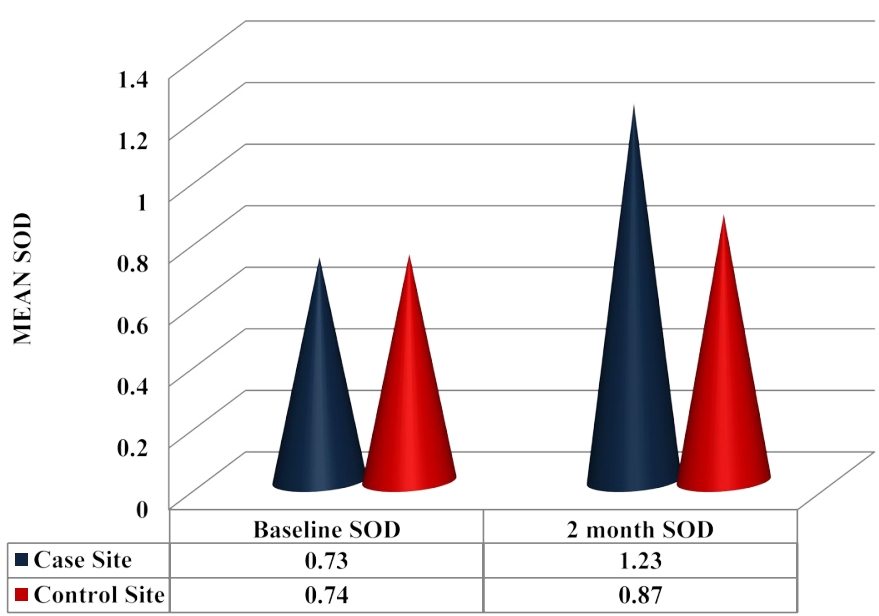

Figure 4. Comparison of Superoxide dismutase between case \& control site among chronic generalized periodontitis patients

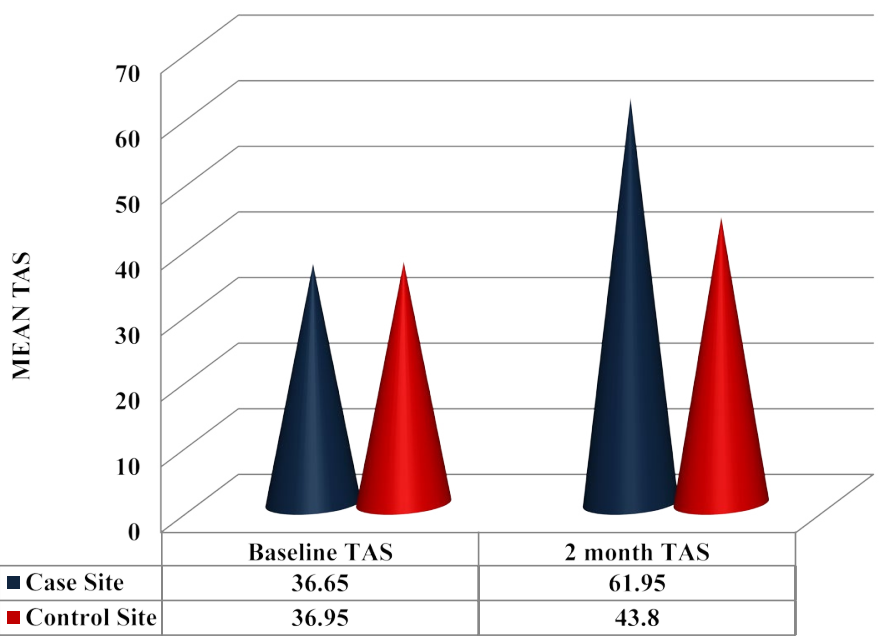

Figure 5. Comparison of Total antioxidant status between case \& control site among chronic generalized periodontitis patients

it increases from $0.73 \pm 0.09$ to $1.23 \pm 0.24$ i.e. $68.49 \%$ increase while among control it increases from $0.74 \pm 0.08$ to $0.87 \pm 0.07$ i.e. $17.56 \%$ increase. After two month there was significantly more incensement in SOD among cases as compare to control and there was statistically significant difference between case and control in SOD $(\mathrm{P}=0.001)$.

Figure 5 reveals Comparison of Total antioxidant status between case \& control site among chronic generalized periodontitis patients. At baseline there was statistically no significant difference in Total antioxidant status between case and control. After two month among case it increases from $36.65 \pm 4.70$ to $61.95 \pm 12.17$ i.e. $69.03 \%$ increase while among control it increases from $36.95 \pm 4.69$ to $43.80 \pm 3.90$ i.e. $18.53 \%$ increase. After two month there was significantly more incensement in TAS among cases as compare to control and there was statistically significant difference between case and control in TAS $(\mathrm{P}=0.001)$.

\section{Discussion}

Aloe vera is a natural product contained in herbal dentifrices with commercial appeal for the control of plaque and gingivitis. Despite its commercial use, this phytotherapic agent does not have sufficient data to support its antigingivitis and antiplaque claims. Therefore, 

study

this study was Evaluation of local - delivery system containing $80 \%$ aloevera gel used as an adjunct to scaling and root planning in chronic Periodontitis and to compare the antioxidant level before and after aloe- Vera application.

Present study explains that Probing pocket depth, After two month among case reduced when compared to control side. This is because aloevera reduces edema of the soft tissues and consequently reduces the bleeding of the gums and exhibits strong antiseptic action in gingival pockets where normal cleaning is difficult $[16,17]$. The results of the present study are similar to the studies by Harjit Kaur Virdi, (2012) [18] on Effect of locally delivered aloe Vera gel as an adjunct to scaling and root planning in the treatment of chronic periodontitis.

The Clinical Attachment loss between case \& control, after two month among case it reduced. This is due to Aloe vera inhibits the production of free oxygen radicals by activated Polymorpho Nuclear Leucocytes (PMNs). It has also shown to provide relief in swelling, bleeding gums and is an antiseptic for pockets and antifungal [19]. The results of this study were in agreement with those presented by Virdi et al [20] The results of the present study are similar to the studies by Surekha rathod et al [21] on clinical efficacy of aloevera chip as an adjunct to non-surgical therapy in the treatment of chronic periodontitis. Clinical attachment loss show significant difference after alovera therapy.

Present study explains that sulcus bleeding index between case \& control site among chronic generalized periodontitis patients. After two month among case it reduced. The result of the present study is similar to the studies by Neha ajmera et al [22] on Aloe Vera: its effect on gingivitis. There is suggestion of a reduction in sulcus bleeding index in all groups in the first month and then in the third month.

In the present study Comparison of Superoxide dismutase between case \& control site among chronic generalized periodontitis patients. At baseline there was statistically no significant difference in Superoxide dismutase between case and control. After two month among case it increased. The results of this study were in agreement with those presented by Sang-Chul Kim et al [23].

Total antioxidant status between case \& control site among chronic generalized periodontitis patients. At baseline there was statistically no significant difference in Total antioxidant status between case and control. After two month among case it increased. Vitamin C present in Aloe vera is involved in collagen synthesis, increases concentration of oxygen at the wound site because of dilation of blood vessels [24]. This study similar with the Sang-Chul Kim et al [23] on Antioxidant profile of whole saliva after scaling and root planing in periodontal disease.

Aloe vera can be applied topically, is easily available, safe to use, cost effective, non-invasive and effective treatment modality for periodontitis.

\section{Conclusion}

The results presented in this study suggest that the aloe Vera gel is effective in the treatment of chronic periodontitis when used as an adjunct to scaling and root planning. It can become an important part of the preventive and therapeutic treatments available for the periodontal diseases. However, due to the limited patient number in this study and a shorter time period further research based on long term studies with larger number of patients and microbial studies is required in this field.

\section{Conflicts of interest}

The authors declare that there is no conflicts of interest regarding the publication of this paper.

\section{References}

1. Kornman KS, Löe H (1993) The role of local factors in the etiology of periodontal diseases. Periodontol 2000 2: 83-97.

2. Novak MJ, Novak KF (2010) Chronic periodontitis. In Newman MG, Takei HH, Klokkevold PR, Carranza FA (eds). Carranza's clinical periodontology. St.louis, Missouni: Saunders Elsevier, 494.

3. Axelsson P, Lindhe $\mathrm{J}$ (1981) The significance of maintenance care in the treatment of periodontal disease. J Clin Periodontol 8(4): 281-294.

4. McNabb H, Mombelli A, Lang NP (1992) Supragingival cleaning 3 times a week. The microbiological effects in moderately deep pockets. J Clin Periodontol 19(5): 348-356.

5. Nogueira-Filho GR, Toledo S, Cury JA (2000) Effect of 3 dentifrices containing triclosan and various additives. An experimental gingivitis study. J Clin Periodontol 27: 494-498.

6. Yates R, Jenkins S, Newcombe R, Wade W, Moran J, Addy M (1993) A 6-month home usage trial of a $1 \%$ chlorhexidine toothpaste (1). Effects on plaque, gingivitis, calculus and toothstaining. J Clin Periodontol 20(2): 130-138.

7. Lee SS, Zhang W, Li Y (2004) The antimicrobial potential of 14 natural herba dentifrices: results of an in vitro diffusion method study. J Am Dent Assoc 135(8): 1133-1141.

8. Botelho MA, Nogueira NA, Bastos GM, Fonseca SG, Lemos TL, et al. (2007) Antimicrobial activity of the essential oil from Lippia sidoides, carvacrol and thymol against oral pathogens. Braz J Med Biol Res 40(3): 349-356.

9. Grindlay D, Reynolds T (1986) The Aloe vera phenomenon: a review of the properties and modern uses of the leaf parenchyma gel. J Ethnopharmacol 16(2-3): 117-151.

10. Shelton RM (1991) Aloe vera. Its chemical and therapeutic properties. Int J Dermatol 30: $679-683$.

11. Pandey R, Mishara A (2010) Antibacterial activities of crude extract of Aloe barbadensis to clinically isolated bacterial pathogens. Appl Biochem Biotechnol 160: 1356-1361.

12. Yagi A, Egusa T, Arase M, Tanabe M, Tsuji H (1997) Isolation and characterization of the glycoprotein fraction with a proliferation-promoting activity on human and hamster cells in vitro from Aloe vera gel. Planta Med 63(1): 18-21.

13. Davis RH, Leitner MG, Russo JM, Byrne ME (1989) Anti-inflammatory activity of Aloe vera against a spectrum of irritants. J Am Podiat Med Assoc 79(6): 263-276.

14. Villalobos OJ, Salazar CR, Sảnchez GR (2001) Effect of a mouthwash made of Aloe vera on plaque and gingival inflammation (in Spanish). Acta Odontol Venez 39: 16-24.

15. De Oliveira SM, Torres TC, Pereira SL, Mota OM, Carlos MX (2008) Effect of a dentifrice containing Aloe vera on plaque and gingivitis control. A double-blind clinical study in humans. J Appl Oral Sci 6(4): 293-296.

16. Oosterwaal PJ, Mikx FH, Van’t Hof MA, Renggli HH (1991) Comparison of the antimicrobial effect of the application ofchlorhexidine gel, amine fluoride gel and stannous fluoride gel in debrided periodontal pockets. J Clin Periodontol 18: 245-51.

17. Greenstein G, Polson A (1998) The role of local drug delivery in the management of periodontal disease: A comprehensive review. J Periodontol 69: 507-20.

18. Harjit Kaur Virdi (2012) Effect of locally delivered aloe vera gel as an adjunct to scaling and root planing in the treatment of chronic periodontitis: A clinical study. Indian Journal of Oral Sciences 3(2): 84-89.

19. Aggarwal BB, Prasad S, Reuter S, Kannappan R, Yadev VR, et al. (2011) Identification of novel anti-inflammatory agents from Ayurvedic medicine for prevention of chronic diseases: "reverse pharmacology" and "bedside to bench" approach. Current Drug Targets 12: 1595-1653.

20. Virdi HK, Jain S, Sharma S (2012) Effect of locally delivered aloe Vera gel as an adjunct to scaling and root planning in the treatment of chronic periodontitis: A clinical study. Indian Journal of Oral Sciences 3(2): 84-89.

21. Surekha rahode (2015) Clinical efficacy of aloevera chip as an adjunct to non-surgical therapy in the treatment of chronic periodontitis. International Journal of Research of Ayurveda Pharm 6(4): 516-519.

22. Neha Ajmera (2013) Aloe vera: It's effect on gingivitis. Journal of Indian Society of Periodontology 17(4): 435-438. 
Singh P (2019) Evaluation of local - delivery system containing 80\% aloe vera gel used as an adjunct to scaling and root planning in chronic periodontitis: A clinical study

23. Sang-Chul K (2010) Antioxidant profile of whole saliva after scaling and root planing in periodontal disease. $J$ Periodontal Implant Science 40: 164-171.
24. 2004. Natural herbal dentifrices: results of an in vitro diffusion method study. $J$ Am Dent Assoc 135(8): 1133-1141.

Copyright: ( 02019 Singh P. This is an open-access article distributed under the terms of the Creative Commons Attribution License, which permits unrestricted use, distribution, and reproduction in any medium, provided the original author and source are credited. 